University of Nebraska - Lincoln

DigitalCommons@University of Nebraska - Lincoln

\title{
Peculiar Effect of Mechanical Stress on Polarization Stability in Micrometer-Scale Ferroelectric Capacitors
}

\author{
Alexei Gruverman \\ University of Nebraska-Lincoln, agruverman2@unl.edu \\ J. S. Cross \\ Tokyo Institute of Technology, Meguro-ku, Tokyo 152-8550, Japan \\ W.S. Oates \\ Florida A\&M/Florida State University, Tallahassee, Florida
}

Follow this and additional works at: https://digitalcommons.unl.edu/physicsgruverman

Part of the Physics Commons

Gruverman, Alexei; Cross, J. S.; and Oates, W.S., "Peculiar Effect of Mechanical Stress on Polarization Stability in Micrometer-Scale Ferroelectric Capacitors" (2008). Alexei Gruverman Publications. 50. https://digitalcommons.unl.edu/physicsgruverman/50

This Article is brought to you for free and open access by the Research Papers in Physics and Astronomy at DigitalCommons@University of Nebraska - Lincoln. It has been accepted for inclusion in Alexei Gruverman Publications by an authorized administrator of DigitalCommons@University of Nebraska - Lincoln. 


\title{
Peculiar effect of mechanical stress on polarization stability in micrometer-scale ferroelectric capacitors
}

\author{
A. Gruverman, ${ }^{1, a)}$ J. S. Cross, ${ }^{2}$ and W. S. Oates ${ }^{3}$ \\ ${ }^{1}$ Department of Physics and Astronomy, University of Nebraska, Lincoln, Nebraska 68588-0111, USA \\ ${ }^{2}$ Graduate School of Engineering, Tokyo Institute of Technology, Meguro-ku, Tokyo 152-8550, Japan \\ ${ }^{3}$ Department of Mechanical Engineering, Florida A\&M/Florida State University, Tallahassee, \\ Florida 32306-2870, USA
}

(Received 10 October 2008; accepted 21 November 2008; published online 16 December 2008)

\begin{abstract}
Piezoresponse force microscopy (PFM) has been used to study the polarization stability in micrometer size $\mathrm{Pb}(\mathrm{Zr}, \mathrm{Ti}) \mathrm{O}_{3}$ capacitors. It is shown that the top electrode thickness has a profound effect on the equilibrium polarization state of poled capacitors triggering spontaneous polarization backswitching in the absence of an applied electric field and leading to the formation of an abnormal domain pattern. PFM examination of poled capacitors with thick $(250 \mathrm{~nm})$ top electrodes reveals domain patterns with the central regions always oriented in the direction opposite to the applied field. It is suggested that the driving force behind the observed effect is a transient response to the residual shear stress created by the top electrode in the poled capacitors during field-induced polarization switching. The proposed mechanism is quantified using finite element ferroelectric phase field modeling. The observed effect provides valuable insight into the polarization retention behavior in micrometer size ferroelectric capacitors. (c) 2008 American Institute of Physics.
\end{abstract}

[DOI: $10.1063 / 1.3046734]$

A unique combination of dielectric, piezoelectric, and optical properties puts ferroelectrics among the most important groups of electronic materials attractive for a variety of microelectronic applications. ${ }^{1,2}$ Investigation of polarization reversal processes in ferroelectrics is of fundamental and practical importance for understanding their quasistatic and dynamic behavior. Reduction in the free energy via formation of ferroelectric domain structures gives rise to a profound mechanical stress effect on the physical properties of ferroelectrics. Accommodation of the misfit strain in epitaxial heterostructures can result in the appearance of phases forbidden in bulk samples and significant size dependence on the dielectric and piezoelectric properties. ${ }^{3}$ Numerous attempts have been made to develop a thermodynamic theory that would account for the effect of the mechanical boundary conditions on structural transformations in ferroelectric films. ${ }^{4-8}$ However, up to now almost all of these studies took into account the mechanical boundary conditions only at the film/substrate interface. On the other hand, the effect of the top interface on the polarization state has largely been ignored. Furthermore, due to the pronounced size effect exhibited by ferroelectrics, ${ }^{9-14}$ scaling of ferroelectric structures brings about an additional aspect to their static and dynamic behavior that needs to be thoroughly understood.

Generally, the polarization state in ferroelectric heterostructures, determined by the thermodynamic minimum of free energy, is a function of electrical and mechanical boundary conditions and depends on the film defect structure, thickness, crystallographic orientation, lattice misfit strain, electrode material, poling conditions, etc. Application of advanced characterization techniques, primarily piezoresponse force microscopy (PFM) ${ }^{15-18}$ has provided additional insight into the microscopic mechanisms of electrically induced transformations in ferroelectric structures.

${ }^{a)}$ Electronic mail: alexei_gruverman@unl.edu.
In this letter, we report on the observation of the mechanical stress effect imposed by the top electrode (TEL) on polarization stability in micrometer-scale $\mathrm{Pb}(\mathrm{Zr}, \mathrm{Ti}) \mathrm{O}_{3}(\mathrm{PZT})$ capacitors. We show that the TEL drastically affects the polarization stability in these capacitors by triggering spontaneous polarization backswitching leading to the formation of abnormal domain configurations. It is proposed that the driving force behind the observed instability is residual shear stress in the poled capacitor, which increases with an increase in the TEL thickness. The electromechanics governing this behavior is described using nonlinear finite element phase field analysis.

The PFM studies have been performed in the (111)oriented tetragonal PZT capacitors. ${ }^{18}$ The top $\mathrm{IrO}_{2}$ electrodes of several $\mu \mathrm{m}^{2}$ have been produced on the PZT surface by reactive ion etching. PZT capacitors with both 50 -nm-thick TELs and 250-nm-thick TELs have been tested using PFM. Visualization of domain patterns in individual capacitors has been performed by applying an oscillating bias of $0.6 \mathrm{~V}$ (peak to peak) at $10 \mathrm{kHz}$ to the TEL with a conductive probing tip.

Figure 1 shows surface topography, PFM amplitude, and phase images of the poled $1 \times 1.5 \mu \mathrm{m}^{2}$ capacitors with a 50-nm-thick TEL. Capacitors in the upper row have been poled by $-5 \mathrm{~V}, 1 \mathrm{~s}$ voltage pulses applied to the TELs, while

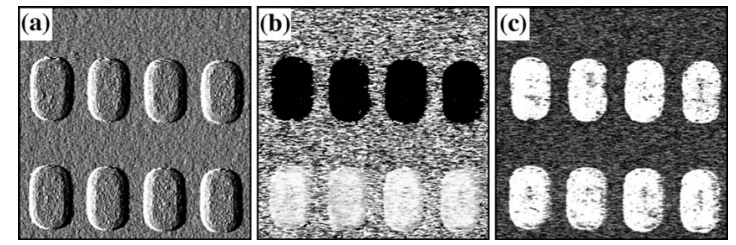

FIG. 1. (a) Topographic, (b) PFM phase, and (c) PFM amplitude of the poled PZT capacitors with 50-nm-thick TELs. Upper row capacitors poled by negative voltage pulses $(-5 \mathrm{~V}, 1 \mathrm{~s})$; bottom row capacitors poled by positive pulses $(+5 \mathrm{~V}, 1 \mathrm{~s})$. The scanning size is $6 \times 6 \mu \mathrm{m}^{2}$. 


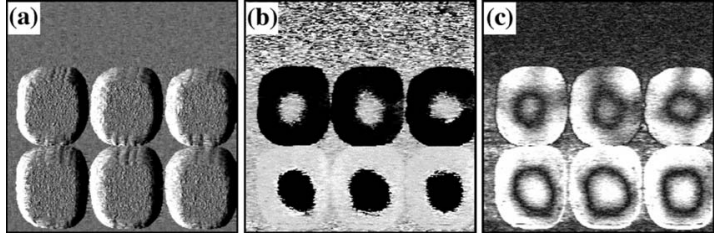

FIG. 2. (a) Topographic, (b) PFM phase, and (c) PFM amplitude of the poled PZT capacitors with 250-nm-thick TELs. Upper row capacitors poled by negative voltage pulses $(-5 \mathrm{~V}, 1 \mathrm{~s})$; bottom row capacitors poled by positive pulses $(+5 \mathrm{~V}, 1 \mathrm{~s})$. The scanning size is $6 \times 6 \mu \mathrm{m}^{2}$.

the bottom row capacitors have been poled into opposite direction by the $+5 \mathrm{~V}, 1 \mathrm{~s}$ voltage pulses. The PFM phase signal, which provides information on the direction of outof-plane polarization, differs by $180^{\circ}$ in the oppositely poled capacitors. The PFM amplitude signal is related to the magnitude of the PFM probe oscillation (due to the piezoelectric response integrated over the capacitor thickness) and is the same for oppositely poled capacitors. Uniform amplitude and phase contrast indicate complete and switching of the capacitors into a stable polarization state.

On the other hand, PFM imaging of the poled capacitors with the 250-nm-thick TEL [Fig. 2(a)] reveals unusual domain patterns. The PFM phase image in Fig. 2(b) shows that after a poling voltage has been turned off, the central regions of the capacitors exhibit polarization opposite to the polarity of the applied voltage. The PFM amplitude signal [Fig. 2(c)] is the same across the domain boundaries in each capacitor, suggesting that the inverse domains in the center extend from the bottom to the TELs of the capacitors. The observed effect is symmetric with respect to the voltage polarity, i.e., the central part of the capacitors always exhibit polarization opposite to the polarity of the applied bias. It should be mentioned that imaging the same capacitors in the PFM mode with an additional dc bias superimposed on the ac imaging voltage resulted in complete switching of polarization in the whole capacitor indicated by a uniform PFM signal across the TEL (not shown here). However, after the dc bias is turned off, the inverse domain in the central region appears again. This behavior is indicative of spontaneous backswitching occurring in the center of the capacitors after application of the poling voltage. This effect has been observed in 250-nm-thick TEL capacitors with the TEL area in the range of several $\mu \mathrm{m}^{2}$ and it gradually disappears in the larger capacitors.

Previously, a similar backswitching effect observed in PZT capacitors with the same crystallographic orientation, composition, and lateral dimensions but with the patterned TEL thickness just slightly above $50 \mathrm{~nm}$ (Ref. 19) has been explained by an isomorphic phase transition in the PZT layer due to a tensile stress induced by a Si substrate. However, our results indicate that the backswitching effect in the PZT capacitors is actually not affected by the substrate but is the result of the presence of a thick $(250 \mathrm{~nm})$ TEL. This apparent contradiction could be explained by the fact that, to overcome a technical problem of contacting the micrometer-scale capacitors, the authors of Ref. 19 additionally deposited $\sim 250$-nm-thick Pt dots of $0.6 \mathrm{~mm}$ in diameter over the electrode-patterned PZT film effectively emulating structures with thick TELs used in our studies. Therefore, as the observed effect is polarization independent (and thus, a built-in field effect can be ruled out as a possible explanation) and is observed only in the capacitors with 250-nm-thick electrodes, we conclude that the anomalous backswitching effect in micrometer PZT capacitors shown in Fig. 2 is induced by the TELs. We suggest that this effect is a result of residual shear stress imposed by a thick TEL.

Before considering the backswitching mechanism let us mention that, generally, the ferroelectric switching behavior is strongly dependent on the crystallographic orientation of the PZT layers. In the (001) orientation, ferroelectric switching is predominantly $180^{\circ}$ polarization reorientation which results in no change in strain. In contrast, $90^{\circ}$ polarization switching, which involves significant strain changes, is energetically favorable in the (111) oriented capacitors. Once the potential is removed, residual elastic energy remains in the film and can potentially provide a mechanical driving force for backswitching.

This effect is simulated using a fully coupled ferroelectric finite element phase field (FEPF) model described as follows. The FEPF model includes mechanical equilibrium, Gauss law, and the time-dependent Ginzburg-Landau equation (see Ref. 20 for details). The three governing equations are obtained by taking the variation in the Gibbs electric energy density:

$$
\begin{aligned}
& \left(\frac{\partial g_{2}}{\partial P_{i, j}}\right)_{, j}-\frac{\partial g_{2}}{\partial P_{i}}=\beta_{i j} \frac{\partial P_{j}}{\partial t}, \\
& \sigma_{j i, j}=0, \\
& D_{i, i}=0,
\end{aligned}
$$

where $g_{2}=\psi-E_{i} D_{i}$ is the electric Gibbs energy density and $\psi$ is the free energy density. The polarization is denoted by $P_{i}$, $\sigma_{i j}$ is the stress, $D_{i}$ is the electric displacement, and $\beta_{i j}$ is a positive definite inverse mobility tensor. Indicial notation has been used where summation applies on repeated indices.

The free energy density used in the finite element model is based on parameters given by Pertsev et al. ${ }^{3}$ for lead titanate. An additional term defining exchange energy via polarization gradients is included in the model and is given in Ref. 21. The backswitching behavior is quantified for the two TEL thicknesses using a two-dimensional analysis on the (111) - ( $\overline{1} \overline{1} 2)$ plane by projecting the $\langle 111\rangle$ orientations onto the (110) plane. In this projection, the polarization component normal to plane (110) (perpendicular to the page) is ignored. The PFM measurements are modeled using the following sets of boundary conditions. Domains in the ferroelectric layer are first allowed to evolve under short-circuit conditions from a quasicubic state with a small random polarization $\left(|\mathbf{P}| \sim 1 \times 10^{-6} \mathrm{C} / \mathrm{m}^{2}\right)$. This results in the formation of an initial (as-grown) domain structure with alternating normal (out-of-plane) polarization components. This polarization state is consistent with PFM observations of the as-grown PZT capacitors.

From this as-grown state, the capacitors of both TEL thicknesses are poled to a state with uniform distribution of the out-of-plane polarization component using the same electric potential. The FEPF simulations show that the presence of a thick TEL causes a large number of twinned domains in the poled capacitor. This effect is illustrated in Fig. 3, which displays maps of the in-plane polarization components in the poled capacitors. While the capacitor with 50-nm-thick TEL 

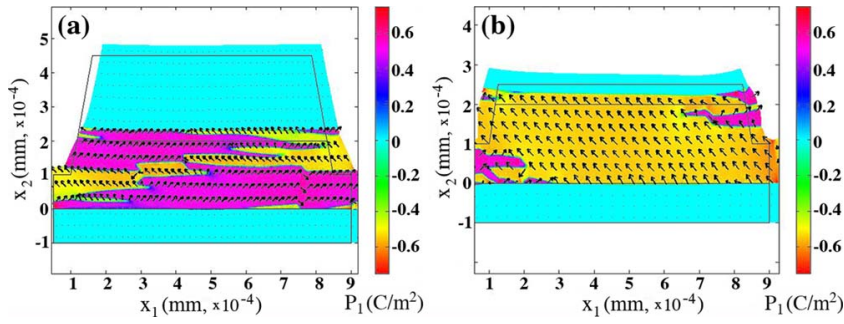

FIG. 3. (Color online) Equilibrium finite element phase field map of the in-plane ( $1 \overline{1} 2)$ polarization component $\left(P_{1}\right)$ in two poled ferroelectric capacitors with a lateral size of $1 \mu \mathrm{m}$ under the applied bias: (a) 250-nm-thick TEL and (b) 50-nm-thick TEL. Similar highly refined finite element mesh densities are used in both models to resolve nanometer size domain wall structures. The polarization vectors are plotted on a coarser length scale for illustrative purposes.

exhibits almost a single domain state, the capacitor with thick TEL shows significant variations in the in-plane polarization due to strong residual stress.

After the external potential is turned off and the capacitors are allowed to relax to equilibrium, a significantly larger amount of polarization switches back in the 250-nm-thick TEL capacitor (Fig. 4). Residual elastic energy is larger in the center away from the stress-free edges which explains why backswitching mainly occurs in the central parts of the capacitors; however, a single center domain is not predicted. This may be due to the $\mathrm{PbTiO}_{3}$ thermodynamic potential used to approximate the $\mathrm{Pb}(\mathrm{Zr}, \mathrm{Ti}) \mathrm{O}_{3}$ composition or possibly a three-dimensional effect. Nevertheless, it should also be noted that this same model correctly predicts the absence of the backswitching effect in the (001)-oriented PZT capacitors in agreement with the experimental results by Stolichnov et al. ${ }^{19}$

This model does not account for flexoelectric effect. ${ }^{22}$ However, strain gradients near the electrode interfaces may lead to higher order ferroelectric switching mechanisms. This may play a role in the capacitor size dependence of the abnormal switching: PFM measurements show that this effect gradually disappears as the lateral dimensions of the capacitor increase. To illustrate the strain gradient effect, consider a boundary value problem consisting of a set of selfequilibrated residual stress loads applied along the surface of an infinitely thick elastic strip of finite width $W$. This boundary value problem approximates the stress at the PZT/TEL interface. The solution to this problem can be represented by a stress vector potential using Stroh's formalism $\varphi$ $=\operatorname{Re}\left\{\mathbf{B}\left\langle e^{-\lambda z_{*}}\right\rangle \mathbf{q}\right\} .{ }^{23}$ Using traction free edges along the lateral edges, the smallest eigenvalue $(\lambda)$ that satisfies equilibrium
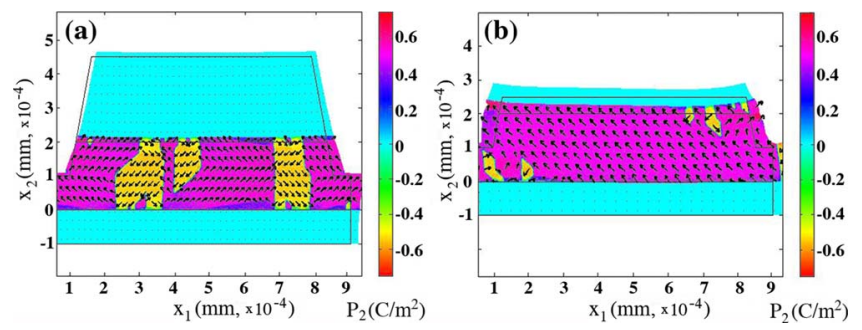

FIG. 4. (Color online) Finite element phase field map of the out-of-plane (111) polarization component $\left(P_{2}\right)$ in two short-circuited ferroelectric capacitors with a lateral size of $1 \mu \mathrm{m}$ under equilibrium conditions: (a) 250-nm-thick TEL and (b) 50-nm-thick TEL. gives the slowest stress decay $\sigma_{i j} \propto e^{-\operatorname{Re}(\lambda) y}$, where $y$ is the distance in the normal direction from the interface. For isotropic materials under antiplane deformation, $\lambda=\pi / W{ }^{23}$ Similar relations are found to exist for in-plane normal or shear loading of an elastic strip of finite thickness. ${ }^{24}$ When the thickness and width are comparable in size, as is the case for a $1 \mu \mathrm{m}^{2}$ capacitor with a $250 \mathrm{~nm}$ TEL, the stress decay rate becomes sensitive to further reductions in the lateral dimension. The stress gradient converges to the exponential relation as the thickness to width ratio becomes very large. This size scaling relation suggests that flexoelectricity may play a role in the backswitching effect. Since the shear strain changes sign upon polarization reversal, the shear strain gradient also changes sign and therefore correlates with the bidirectional spontaneous backswitching behavior.

In summary, we have shown that the TEL thickness has a dramatic effect on equilibrium domain structures in micrometer thin film capacitors. Mechanical constraints are found to create residual shear stresses during ferroelectric switching in films grown in the $\langle 111\rangle$ orientation leading to complex twinned domain structures. The residual elastic energy results in spontaneous polarization backswitching in the central regions of capacitors.

A.G. acknowledges fruitful discussions with A. Tagantsev (EPFL). W.S.O. greatly appreciates discussions with C. Landis (UT-Austin).

${ }^{1}$ J. F. Scott, Science 315, 954 (2007).

${ }^{2}$ O. Auciello, J. F. Scott, and R. Ramesh, Phys. Today 51(7), 22 (1998).

${ }^{3}$ N. A. Pertsev, A. G. Zembilgotov, and A. K. Tagantsev, Phys. Rev. Lett. 80, 1988 (1998).

${ }^{4}$ N. A. Pertsev, G. Arlt, and A. G. Zembilgotov, Microelectron. Eng. 29, 135 (1995).

${ }^{5}$ Y. Yano, K. Iijima, Y. Daitoh, T. Terashima, Y. Bando, Y. Watanabe, H. Kasatani, and H. Terauchi, J. Appl. Phys. 76, 7833 (1994).

${ }^{6}$ J. S. Speck, A. Seifert, W. Pompe, and R. Ramesh, J. Appl. Phys. 76, 477 (1994).

${ }^{7}$ W. Pompe, X. Gong, Z. Suo, and J. S. Speck, J. Appl. Phys. 74, 6012 (1993).

${ }^{8}$ Y. Gao and Z. Suo, J. Appl. Mech. 69, 419 (2002).

${ }^{9}$ M. Dawber, K. M. Rabe, and J. F. Scott, Rev. Mod. Phys. 77, 1083 (2005).

${ }^{10}$ M. M. Saad, P. Baxter, R. M. Bowman, J. M. Gregg, F. D. Morrison, and J. F. Scott, J. Phys.: Condens. Matter 16, L451 (2004).

${ }^{11}$ R. Bruinsma and A. Zangwill, J. Phys. 47, 2055 (1986).

${ }^{12}$ C. S. Ganpule, A. Stanishevsky, S. Aggarwal, J. Melngailis, E. Williams, R. Ramesh, V. Joshi, and C. P. de Araujo, Appl. Phys. Lett. 75, 3874 (1999).

${ }^{13}$ L. Roytburd, S. P. Alpay, V. Nagarajan, C. S. Ganpule, S. Aggarwal, E. D. Williams, and R. Ramesh, Phys. Rev. Lett. 85, 190 (2000).

${ }^{14}$ S. Buhlmann, B. Dwir, J. Baborowski, and P. Muralt, Appl. Phys. Lett. 80, 3195 (2002).

${ }^{15}$ S. Jesse, S. V. Kalinin, R. Proksch, A. P. Baddorf, and B. J. Rodriguez, Nanotechnology 18, 435503 (2007).

${ }^{16}$ S. V. Kalinin and D. A. Bonnell, Phys. Rev. B 65, 125408 (2002).

${ }^{17}$ S. Hong, J. Woo, H. Shin, J. Jeon, Y. E. Pak, E. L. Colla, N. Setter, E. Kim, and K. No, J. Appl. Phys. 89, 1377 (2001).

${ }^{18}$ A. Gruverman, B. J. Rodriguez, R. J. Nemanich, A. I. Kingon, J. S. Cross, and M. Tsukada, Appl. Phys. Lett. 82, 3071 (2003)

${ }^{19}$ I. Stolichnov, E. Colla, A. Tagantsev, S. Bharadwaja, S. Hong, N. Setter, J. S. Cross, and M. Tsukada, Appl. Phys. Lett. 80, 4804 (2002).

${ }^{20}$ Y. Su and C. Landis, J. Mech. Phys. Solids 55, 280 (2007).

${ }^{21}$ The exchange energy is given by $\psi_{G}=1 / 2 a_{0}\left(P_{1,1}^{2}+P_{2,2}^{2}+P_{1,2}^{2}+P_{2,1}^{2}\right)$, where $a_{0}=8 \times 10^{-13} \mathrm{~V} \mathrm{~m}^{3} / \mathrm{C}$.

${ }^{22}$ W. Ma and L. E. Cross, Appl. Phys. Lett. 82, 3293 (2003).

${ }^{23}$ T. Ting, Anisotropic Elasticity (Oxford, Oxford, 1996), pp. 357-364.

${ }^{24} \mathrm{~S}$. Timoshenko and J. Goodier, Theory of Elasticity (McGraw-Hill, New York, 1951), pp. 46-52. 